\title{
Clinical outcomes of a beveled tip, ultra-high speed, 25-gauge pars plana vitrectomy system
}

Harvey S. Uy ${ }^{1,2,3^{*}}$, Vicente Lorenzo O. Cabahug ${ }^{4}$, Jose Carlo M. Artiaga ${ }^{5}$, Pik Sha Chan ${ }^{1,3}$ and Jordan T. Famadico ${ }^{1,6}$

\begin{abstract}
Objective: To report the clinical outcomes of a 25-gauge, beveled-tip, 10,000 cuts-per-minute (cpm) microincisional vitrectomy surgery (MIVS) system.

Methods: Prospective case series of eyes undergoing primary pars plana vitrectomy (PPV) for common vitreoretinal indications. Main outcome measures were: rate of achieving surgical objectives, operative times, number of surgical steps, use of ancillary instruments, corrected distance visual acuity (CDVA), and adverse events (AE).

Results: The surgical objectives were achieved in all eyes. Mean total operative time (TOT), core, shave and total vitrectomy times were $1891 \pm 890,204 \pm 120,330 \pm 320,534 \pm 389$ s, respectively. Mean number of surgical steps was 4.3 \pm 1.5. Mean number of ancillary instruments used was $4.5 \pm 1.9$. Mean CDVA improved by $0.53 \pm 0.56$ logMAR units $(P<0.001) 3$ months postoperatively. AE included elevated IOP (8\%), hypotony $(6 \%)$, and re-detachment $(2 \%)$. Majority (82\%) had no postoperative discomfort. The number of surgical steps demonstrated a positive correlation with TOT ( $p<0.05)$, number of ancillary instruments used $(p<0.05)$, and postoperative Day 1 IOP $(p<0.05)$. The number of times ancillary instrumentation was used demonstrated a positive correlation with TOT $(p<0.05)$.
\end{abstract}

Conclusion: Beveled-tip, 10,000 cpm MIVS system effectively and safely performs common VR procedures of varying complexity and may reduce operative times and use of ancillary instrumentation.

Keywords: Pars plana vitrectomy, Microincisional vitrectomy surgery, MIVS, Ultra-high speed vitrectomy, Beveled tip cutter probe

\section{Introduction}

In the 1970's, Robert Machemer performed the first closed-system, pars plana vitrectomy (PPV) using a single-port, 17-gauge (17G) system with a maximal cut rate of 400 cuts per minute (cpm) [1]. Since then, the efficacy, efficiency and safety of PPV has improved with the introduction of microincisional vitrectomy surgery (MIVS), wide-angle viewing as well as new surgical techniques such as membrane dissection, internal subretinal fluid

*Correspondence: harveyuy@yahoo.com

2 Department of Ophthalmology and Visual Sciences, University of the Philippines, Manila, Philippines

Full list of author information is available at the end of the article drainage, fluid-air exchange (FAX), and endophotocoagulation [2-5].

Smaller gauge probes, faster cut rates and fluidics control have increased PPV precision, decreased operative times, hastened postoperative recovery and reduced postoperative pain and complications [6, 7]. A recently introduced beveled-tip cutter probe (BTCP) with shortened port-tip distance potentially facilitates access to surgical tissue planes, permits an expanded range of surgical maneuvers and performs multiple functions (Fig. 1) $[4,5]$. There are few publications describing the clinical use of this relatively novel cutter probe configuration; fewer still are publications that report on the multifunctional capabilities of cutter probes and how they might potentially enhance efficiency by reducing the usage of 


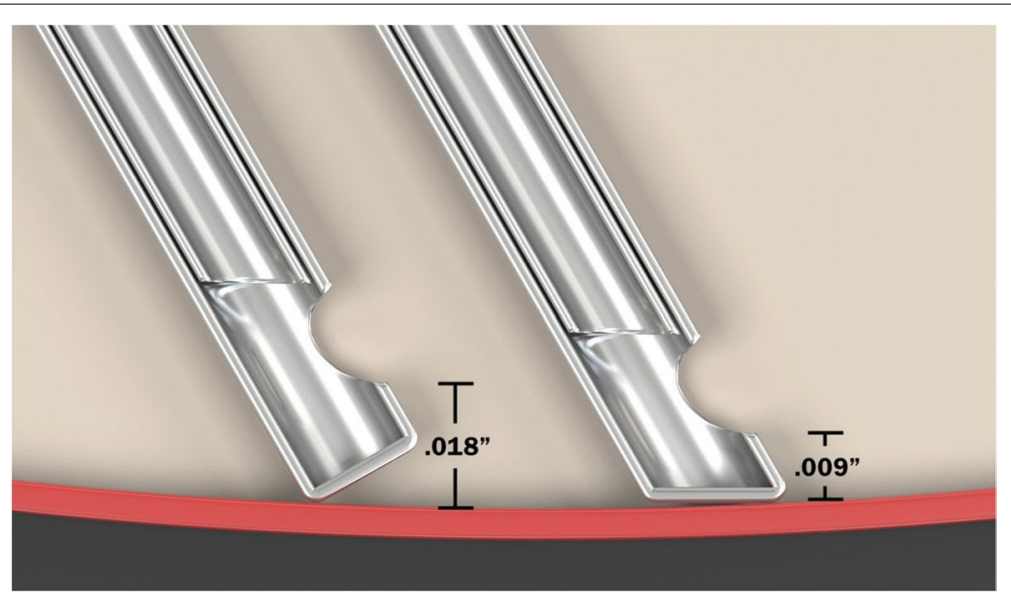

Fig. 1 Schematic of the Beveled probe (right) compared with the conventional probe design (Alcon Laboratories, Fort Worth, TX, USA)

ancillary instrumentation. The purpose of this study was to describe the clinical outcomes and assess the utility of using a 25 -gauge (25G), $10,000 \mathrm{cpm} \mathrm{BTCP}$ for the treatment of various vitreoretinal (VR) diseases.

\section{Patients and methods}

This is a single-center, prospective case series of 50 eyes that underwent primary PPV performed at the Peregrine Eye and Laser Institute (PELI) from April 24 to October 24,2019 . The study was conducted according to the tenets of the Declaration of Helsinki. The study protocol and informed consent form was approved by the PELI-Institutional Review Board. Participants provided informed consent prior to enrollment. We included eyes undergoing primary PPV for non-resolving vitreous hemorrhage $(\mathrm{VH})$ and vitritis, clinically significant epiretinal membranes (ERM), clinically significant lamellar and full thickness macular holes $(\mathrm{MH})$, vitreo-macular traction (VMT), rhegmatogenous retinal detachment (RRD) or tractional retinal detachment (TRD), retained lens fragments (RLF), and dislocated intraocular lenses (DIS). We excluded eyes with history of glaucoma, prior glaucoma surgery, scleral thinning, recent ( $<3$ months) ocular infection, central corneal opacification which would affect surgical visualization, clinically significant crystalline lens opacities where the patient did not agree to simultaneous lens removal, non-dilating pupils $(<5 \mathrm{~mm}$ with maximal dilation) and choroidal detachment.

The patients underwent comprehensive ophthalmologic assessment including corrected distance visual acuity (CDVA), intraocular pressure (IOP) measurement, slit-lamp and dilated fundus examination at all visits. IOP was measured using applanation tonometry during clinic visits and digital contact tonometry during surgery (Tonopen AVIA, Reichert Technologies, Depew, NY, USA).
Elevated IOP was defined as $>22 \mathrm{mmHg}$ while hypotony was defined as $<5 \mathrm{mmHg}$.

PPV was performed using 25G, 10,000 cpm BTCP (25G Advanced Ultravit, CONSTELLATION Vision System, Alcon Surgical, Irvine, CA, USA) by a trained retinal surgeon (HSU, PCU or JTF). A wide-angle viewing system (Resight 700, Zeiss Medical Technology, Jena, Germany) and high magnification contact lens (HR Direct High Mag Surgical Lens, Volk Optical, Inc., Mentor, $\mathrm{OH}, \mathrm{USA}$ ) provided surgical visualization. Trocars were inserted $3.5 \mathrm{~mm}$ from the limbus. Central vitreous was removed using the core vitrectomy setting (open-biased, $\mathrm{IOP}=25 \mathrm{mmHg}$, maximum vacuum $=650 \mathrm{mmHg}$, cut-rate $=10,000 \mathrm{cpm})$; peripheral vitreous was removed using shave vitrectomy setting (closed-biased, $\mathrm{IOP}=25 \mathrm{mmHg}$, maximum vacuum $=300 \mathrm{mmHg}$, cut rate $=10,000 \mathrm{cpm}$ ).

For membrane peeling, visualization was enhanced using Brilliant Blue, Trypan Blue and soluble lutein (Doubledyne, Horus Pharma, St. Laurent du Var, France) which was refluxed onto the retinal surface using the BTCP. Initial ERM or internal limiting membrane (ILM) flap edges were created using ILM forceps or nitinol loops. Whenever safely possible, the BTCP was used to grasp the flap edge to perform membrane removal, otherwise forceps was used to complete the process.

For RRD, the 25G BTCP was used to aspirate subretinal fluid through extramacular pre-existing breaks or drainage retinotomies. Small residual amounts of fluid on the macular or optic nerve head surface were aspirated using a soft-tip, backflush handpiece (25-gauge, Grieshaber Advanced Backflush DSP, Alcon Surgical, Fort Worth, TX, USA). Endophotocoagulation was applied around the retinal breaks and in cerclage 
fashion along the equatorial region followed by placement of tamponade agents. The patients were typically followed up on postoperative Day 1, Week 1, Month 1 and Month 3.

The main outcome measures were rate of achievement of surgical objectives, total operative time (TOT) from first trocar insertion to last trocar removal, core vitrectomy time (CVT), shave vitrectomy time (SVT), total vitrectomy time $(\mathrm{TVT}=\mathrm{CVT}+\mathrm{SVT})$ as recorded by the circulating nurse using a stopwatch, perioperative IOP, number of surgical steps, number of times ancillary instrumentation was placed into the eye, CDVA, and adverse events (AE). The number of surgical steps referred to the number of maneuvers that were executed: PPV, ERM or ILM peeling, FAX, endolaser treatment, gas or silicon oil tamponade, amniotic membrane application, lens material or IOL removal, and secondary IOL implantation. Pain at Postoperative Day 1 was reported by the patient using the following ordinal scale: 0 - no pain; 1 - mild pain not requiring medication; 2 - moderate pain less than half of waking day requiring medication; 3 - moderate pain more than half of waking day requiring medication; 4 - pain that is interrupting sleep and requiring medication.

Descriptive analysis was used for continuous and interval variables. Correlation analysis was also applied, where Pearson $r$ coefficient was applied for interval level of data, while Spearman $r$ rank coefficient for ordinal level of data such as pain scores. Level of significance is at $5 \%$. Medcalc Statistical software version 19.2.1 was utilized for statistical calculations.

\section{Results}

Fifty consecutive eyes underwent PPV for various indications (Table 1). The mean patient age was $57.2 \pm 13.5$ (range, 16-84). Baseline CDVA ranged from 20/20 to light perception vision with a mean CDVA of $1.24 \pm 0.88$ $\operatorname{logMAR}$ units. The mean preoperative IOP was $13.2 \pm 4.2$ (range, $4-30$ ) $\mathrm{mmHg}$. The surgical indications were $\mathrm{MH}(22 \%)$, RRD (20\%), vitreous hemorrhage (20\%), ERM (12\%), TRD (10\%), DIS (6\%), vitritis (6\%) and RLF (4\%). All RRD eyes were macula-off and all TRD eyes had foveal involvement as confirmed by optical coherence tomography. Thirteen (26\%) had multiple surgical indications: $\mathrm{MH}+\mathrm{ERM}(8 \%), \mathrm{VH}+\mathrm{ERM}(6 \%), \mathrm{TRD}+\mathrm{VH}$ (4\%), TRD + Vitritis (2\%), RRD + VH (2\%), dislocated $\mathrm{IOL}+\mathrm{VH}(2 \%)$ and $\mathrm{VH}+$ retinal tear $(2 \%)$.

The surgical objectives were attained in all eyes. At the 3-month postoperative visit, the mean CDVA improved $(P<0.05)$ from 1.24 to 0.71 logMAR units. CDVA improved by 2 lines or more in $56 \%$, remained unchanged in $40 \%$, and decreased by 2 lines or more in $4 \%$ of eyes.
The mean TOT was $1891 \pm 890$ (range, $510-3930$ ) seconds. The mean CVT, SVT, and TVT were 204 \pm 120 (range, 60-594), $330 \pm 320$ (range, 90-1782) and $534 \pm 389$ (range, 155-2376) seconds, respectively. The mean number of surgical steps was $4.3 \pm 1.5$ (range, 1-9); the mean number of times ancillary instruments were placed in each eye was $4.5 \pm 1.9$ (range, 1-8) times.

Intraoperative $\mathrm{AE}$ included an iatrogenic retinal break in one eye $(2 \%)$ and nicked retinal vessel in another eye (2\%) which was easily controlled by increasing IOP. Postoperative AE included IOP elevation in 4 eyes $(8 \%)$, hypotony in 3 eyes (6\%) and recurrent RRD, in one eye with long axial length (2\%). None of the eyes required sclerotomy suturing.

PPV using the 25G BTCP was well-tolerated. The mean postoperative Day 1 pain grading was $0.2 \pm 0.5$ (range, $0-2)$. Forty-one patients $(82 \%)$ reported no pain, $2(4 \%)$ reported mild pain, and 1 patient (2\%) reported moderate pain.

The number of surgical steps demonstrated a positive correlation with TOT $(p<0.05)$, number of ancillary instruments used $(p<0.05)$, and postoperative Day 1 IOP $(p<0.05)$. The number of times ancillary instrumentation was used demonstrated a positive correlation with TOT $(p<0.05)$. Postoperative day 1 IOP was not correlated to TOT, number of ancillary instruments used, nor to CVT or SVT. Postoperative pain scores and CDVA change after 3 months were unrelated to other variables (Table 2).

\section{Discussion}

MIVS, or transconjunctival sutureless vitrectomy surgery as first described by Fujii and colleagues, has become the standard of care for VR surgery [6, 7]. Incremental technological improvements such as higher cutting speeds, better fluidics, and cutter probe modifications such as the beveled-tip design used in this study, continue to enhance the effectiveness and safety of PPV. This open-label, prospective case series demonstrated that a high speed, 25G, BTCP as utilized by multiple surgeons effectively and safely achieved the surgical objectives for common VR conditions. Additionally, this study uniquely explored the relationship of surgical efficiency parameters such as operative time, usage of ancillary instrumentation and their relationship to surgical complexity.

Using this system, we observed significant visual acuity improvement of $0.53 \pm 0.56 \log$ MAR units $(18.36 \pm 19.61$ ETDRS letters, $P<0.001) 3$ months after surgery. The magnitude of improvement in postoperative visual outcomes observed here is comparable to results of studies using similar gauge instrumentation [8-13]. Mitsui et al. prospectively compared $27 \mathrm{G}$ and $25 \mathrm{G}$ vitrectomy systems for eyes with ERM and measured visual acuity 
Table 1 Beveled tip, ultra-high speed, 25-gauge pars plana vitrectomy system study data

\begin{tabular}{|c|c|c|c|c|c|c|c|c|c|}
\hline Patient & Age (yrs) & Gender & Diagnosis & Surgical steps & \# Steps & Core time (secs) & $\begin{array}{l}\text { Shave } \\
\text { time (secs) }\end{array}$ & PPV time (secs) & $\begin{array}{l}\text { Total } \\
\text { time (secs) }\end{array}$ \\
\hline 1 & 29 & M & $\mathrm{VH}$ & PPV, LX & 2 & 186 & 180 & 366 & 756 \\
\hline 2 & 69 & $\mathrm{~F}$ & $\mathrm{MH}+\mathrm{ERM}$ & PPV, ERM, ILM, LX, FAX, GT & 6 & 372 & 177 & 549 & 2130 \\
\hline 3 & 56 & M & RRD & PPV, FAX, LX, GT & 4 & 474 & 612 & 1086 & 2526 \\
\hline 4 & 62 & M & RRD & PPV, FAX, LX, GT & 4 & 300 & 708 & 1008 & 1956 \\
\hline 5 & 61 & $\mathrm{~F}$ & $\mathrm{VH}$ & PPV, LX & 2 & 240 & 360 & 600 & 1350 \\
\hline 6 & 57 & $\mathrm{~F}$ & RRD & PPV, FAX, LX, GT & 4 & 120 & 978 & 1098 & 2676 \\
\hline 7 & 64 & M & $\mathrm{MH}+\mathrm{ERM}$ & PPV, ERM, ILM, FAX, LX, GT & 6 & 270 & 549 & 819 & 3636 \\
\hline 8 & 34 & M & RRD & PPV, FAX, LX, ST & 4 & 246 & 882 & 1128 & 3078 \\
\hline 9 & 38 & M & $\mathrm{VH}+\mathrm{ERM}$ & PPV, ERM, FAX, LX & 4 & 234 & 296 & 530 & 1506 \\
\hline 10 & 69 & M & $\mathrm{MH}$ & PPV, ERM, ILM, FAX, LX, GT, AM & 7 & 474 & 416 & 890 & 3786 \\
\hline 11 & 55 & $\mathrm{~F}$ & $\mathrm{VH}+\mathrm{ERM}$ & PE, PPV, ERM, LX, FAX & 5 & 246 & 302 & 548 & 1920 \\
\hline 12 & 74 & $\mathrm{~F}$ & $\mathrm{DIS}+\mathrm{VH}$ & PPV, LX, RIOL & 3 & 120 & 120 & 240 & 1686 \\
\hline 13 & 53 & $\mathrm{~F}$ & $\mathrm{TRD}+\mathrm{VH}$ & PE, PPV, ERM, LX, FAX, LX & 6 & 594 & 1782 & 2376 & 3726 \\
\hline 14 & 52 & M & $\mathrm{TRD}+\mathrm{VH}$ & $P E, P P V, E R M$ & 3 & 474 & 119 & 593 & 2250 \\
\hline 15 & 59 & M & $\mathrm{RRD}+\mathrm{VH}$ & PPV, ERM, FAX, LX & 4 & 180 & 183 & 363 & 810 \\
\hline 16 & 54 & M & MH, ERM & PPV, ERM, FAX, LX & 4 & 120 & 180 & 300 & 1890 \\
\hline 17 & 16 & $\mathrm{~F}$ & RRD & PPV, ERM, FAX, LX, GT & 5 & 180 & 400 & 580 & 3930 \\
\hline 18 & 63 & M & $\mathrm{VH}+\mathrm{ERM}$ & PPV, ERM, FAX, LX & 4 & 120 & 120 & 240 & 1230 \\
\hline 19 & 77 & $\mathrm{~F}$ & ERM & PPV, ERM, LX, FAX & 4 & 180 & 312 & 492 & 1398 \\
\hline 20 & 63 & M & $\mathrm{VH}$ & PPV, LX & 2 & 297 & 408 & 705 & 1140 \\
\hline 21 & 84 & $\mathrm{~F}$ & VIT & PPV, LX & 2 & 238 & 246 & 484 & 798 \\
\hline 22 & 42 & M & DIS & PPV, LX, RIOL, SIOL & 4 & 241 & 180 & 421 & 2118 \\
\hline 23 & 70 & M & $\mathrm{VH}+\mathrm{RT}$ & PPV, LX, FAX & 3 & 180 & 234 & 414 & 768 \\
\hline 24 & 61 & $\mathrm{~F}$ & ERM & PPV, ERM, LX, FAX & 5 & 121 & 120 & 241 & 1446 \\
\hline 25 & 69 & $\mathrm{~F}$ & ERM & PPV, ERM, LX, FAX & 4 & 119 & 121 & 239 & 1026 \\
\hline 26 & 74 & M & RLF & PPV & 1 & 293 & 120 & 413 & 510 \\
\hline 27 & 60 & M & RRD & PPV, FAX, LX, GT & 4 & 120 & 1188 & 1308 & 2640 \\
\hline 28 & 47 & $\mathrm{~F}$ & $\mathrm{VH}, \mathrm{TRD}$ & PPV, ERM, ILM, FAX, LX, GT & 6 & 236 & 426 & 662 & 3420 \\
\hline 29 & 84 & $\mathrm{~F}$ & $\mathrm{MH}, \mathrm{ERM}$ & PPV, ERM, ILM, FAX, LX, GT & 6 & 60 & 240 & 300 & 2130 \\
\hline 30 & 56 & M & RRD & PPV, PE, PCIOL, ERM, FAX, LX, GT & 7 & 359 & 546 & 905 & 2682 \\
\hline 31 & 34 & M & $\mathrm{TRD}+\mathrm{VIT}$ & PPV, PE, PCIOL, LX & 4 & 240 & 300 & 540 & 708 \\
\hline 32 & 63 & M & RRD & PPV, FAX, LX, GT & 4 & 240 & 492 & 732 & 1668 \\
\hline 33 & 64 & $\mathrm{~F}$ & $\mathrm{MH}$ & PPV, ERM, LX, FAX & 4 & 120 & 240 & 360 & 2676 \\
\hline 34 & 52 & $\mathrm{~F}$ & VIT & PPV, MD, FAX & 3 & 301 & 240 & 541 & 1686 \\
\hline 35 & 56 & M & ERM & PPV, ERM, LX, FAX & 4 & 120 & 120 & 240 & 1728 \\
\hline 36 & 59 & M & DIS & PPV, LX, RIOL & 3 & 90 & 90 & 180 & 2010 \\
\hline 37 & 62 & $\mathrm{~F}$ & $\mathrm{MH}$ & $\begin{array}{l}\text { PPV, PE, PCIOL, ERM, ILM, FAX, LX, } \\
\text { AM, GT }\end{array}$ & 9 & 105 & 360 & 465 & 2286 \\
\hline 38 & 38 & $\mathrm{~F}$ & $\mathrm{VH}$ & PPV, MD, FAX & 3 & 138 & 144 & 282 & 768 \\
\hline 39 & 51 & $\mathrm{~F}$ & $\mathrm{MH}$ & PPV, ERM, ILM, FAX, LX & 5 & 202 & 120 & 322 & 1116 \\
\hline 40 & 66 & $\mathrm{~F}$ & $\mathrm{MH}$ & PPV, ERM, ILM, FAX, LX, GT & 6 & 150 & 180 & 330 & 2076 \\
\hline 41 & 62 & $\mathrm{~F}$ & RRD & PPV, FAX, LX, GT & 4 & 83 & 589 & 672 & 2190 \\
\hline 42 & 64 & M & ERM & PPV, ERM, ILM, FAX & 4 & 120 & 120 & 240 & 1308 \\
\hline 43 & 65 & M & $\mathrm{MH}$ & PPV, ERM, ILM, FAX, LX & 5 & 72 & 165 & 237 & 1866 \\
\hline 44 & 44 & M & $\mathrm{VH}$ & PPV, ERM, LX, FAX & 4 & 90 & 120 & 210 & 918 \\
\hline 45 & 63 & $\mathrm{~F}$ & ERM & PPV, ERM, ILM, FAX, LX & 5 & 120 & 120 & 240 & 1140 \\
\hline 46 & 38 & M & RLF & PPV, LX & 2 & 60 & 95 & 155 & 1425 \\
\hline 47 & 55 & $\mathrm{~F}$ & TRD & PPV, PE, PCIOL, ERM, LX, FAX & 6 & 180 & 150 & 330 & 1758 \\
\hline 48 & 62 & $\mathrm{~F}$ & $\mathrm{MH}$ & PPV, ERM, ILM, FAX, LX & 5 & 120 & 120 & 240 & 2250 \\
\hline
\end{tabular}


Table 1 (continued)

\begin{tabular}{|c|c|c|c|c|c|c|c|c|c|c|c|c|c|}
\hline Patient & Age (yrs) & Gender & Diagn & osis & Surgica & steps & & \# Steps & Core tir & ne (secs) & $\begin{array}{l}\text { Shave } \\
\text { time (secs) }\end{array}$ & PPV time (secs) & $\begin{array}{l}\text { Total } \\
\text { time (secs) }\end{array}$ \\
\hline 49 & 52 & M & $\mathrm{VH}$ & & PPV, LX, & EAX & 3 & 3 & 60 & & 120 & 180 & 930 \\
\hline 50 & 56 & M & TRD + & VH & $P P V, P E$ & $\mathrm{CIOL}, \mathrm{LX}, \mathrm{ER}$ & FAX & 6 & 180 & & 120 & 300 & 3120 \\
\hline Mean & 57.2 & & & & & & & 4.3 & 204 & & 330 & 534 & 1891 \\
\hline SD & 13.5 & & & & & & & 1.5 & 120 & & 320 & 389 & 890 \\
\hline Patient & IOP Day 1 & $\begin{array}{l}\text { \#Times } \\
\text { ancillary Instr } \\
\text { placed in eye }\end{array}$ & rument & objecti & tive achieved? & Wound leak? & Pain score (0-4) & Preoper & rative DCVA & Month 3 DCVA & /A $\quad$ logmAR Cha & Adverse Events & Comments \\
\hline 1 & 4 & 2 & & Y & & N & 0 & 1.854 & & 0.398 & -1.456 & N & \\
\hline 2 & 8 & 8 & & Y & & $\mathrm{N}$ & 0 & 2.301 & & 1.000 & -1.301 & $\mathrm{~N}$ & \\
\hline 3 & 18 & 4 & & Y & & N & 0 & 2.602 & & 2.301 & -0.301 & $\mathrm{~N}$ & \\
\hline 4 & 10 & 3 & & Y & & $\mathrm{N}$ & 0 & 2.602 & & 1.301 & -1.301 & $\mathrm{~N}$ & \\
\hline 5 & 8 & 2 & & Y & & N & 0 & 1.854 & & 0.398 & -1.456 & $\mathrm{~N}$ & \\
\hline 6 & 18 & 3 & & Y & & N & 0 & 2.301 & & 1.301 & -1.000 & Y & $\begin{array}{l}\text { Redetach- } \\
\text { ment }\end{array}$ \\
\hline 7 & 10 & 8 & & Y & & $\mathrm{N}$ & 0 & 2.301 & & 1.000 & -1.301 & $\mathrm{~N}$ & \\
\hline 8 & 6 & 7 & & Y & & $\mathrm{N}$ & 0 & 1.854 & & 1.301 & -0.553 & $\mathrm{~N}$ & \\
\hline 9 & 17 & 5 & & Y & & N & 0 & 0.155 & & 0.155 & 0.000 & N & \\
\hline 10 & 8 & 7 & & Y & & N & 0 & 0.824 & & 0.097 & -0.727 & y & $\begin{array}{l}\text { Nicked } \\
\text { retinal blood } \\
\text { vessel }\end{array}$ \\
\hline 11 & 4 & 4 & & Y & & $\mathrm{N}$ & 1 & 0.699 & & 0.699 & 0.000 & $\mathrm{~N}$ & \\
\hline 12 & 7 & 4 & & Y & & N & 2 & 0.301 & & 0.301 & 0.000 & N & \\
\hline 13 & 19 & 4 & & Y & & $\mathrm{N}$ & 1 & 1.854 & & 1.301 & -0.553 & $\mathrm{~N}$ & \\
\hline 14 & 10 & 5 & & Y & & $\mathrm{N}$ & 1 & 1.854 & & 1.301 & -0.553 & $\mathrm{~N}$ & \\
\hline 15 & 4 & 3 & & Y & & N & 0 & 1.854 & & 0.155 & -1.699 & N & \\
\hline 16 & 16 & 6 & & Y & & $\mathrm{N}$ & 0 & 0.000 & & 0.000 & 0.000 & N & \\
\hline 17 & 18 & 7 & & Y & & N & 0 & 2.602 & & 0.699 & -1.903 & $\mathrm{~N}$ & \\
\hline 18 & 12 & 4 & & y & & $\mathrm{N}$ & 0 & 1.301 & & 0.301 & -1.000 & $\mathrm{~N}$ & \\
\hline 19 & 0 & 6 & & Y & & $\mathrm{N}$ & 0 & 0.301 & & 0.301 & 0.000 & Y & Hypotony \\
\hline 20 & 20 & 6 & & Y & & $\mathrm{N}$ & 1 & 1.854 & & 1.000 & -0.854 & N & \\
\hline 21 & 4 & 2 & & Y & & $\mathrm{N}$ & 1 & 1.854 & & 0.699 & -1.155 & $\mathrm{~N}$ & \\
\hline 22 & 8 & 3 & & Y & & N & 0 & 0.301 & & 0.000 & -0.301 & $\mathrm{~N}$ & \\
\hline 23 & 14 & 2 & & Y & & $\mathrm{N}$ & 0 & 2.602 & & 1.000 & -1.602 & $\mathrm{~N}$ & \\
\hline 24 & 9 & 7 & & Y & & $\mathrm{N}$ & 0 & 0.301 & & 0.155 & -0.146 & $\mathrm{~N}$ & \\
\hline 25 & 4 & 4 & & Y & & $\mathrm{N}$ & 0 & 0.174 & & 0.097 & -0.077 & $\mathrm{~N}$ & \\
\hline 26 & 8 & 4 & & Y & & $\mathrm{N}$ & 0 & 0.174 & & 0.000 & -0.174 & $\mathrm{~N}$ & \\
\hline 27 & 4 & 3 & & Y & & $\mathrm{N}$ & 0 & 1.301 & & 0.699 & -0.602 & $\mathrm{~N}$ & \\
\hline 28 & 4 & 5 & & Y & & $\mathrm{N}$ & 0 & 2.301 & & 1.301 & -1.000 & Y & $\begin{array}{l}\text { IOP eleva- } \\
\text { tion }\end{array}$ \\
\hline 29 & 27 & 5 & & Y & & $\mathrm{N}$ & 0 & 0.699 & & 0.824 & 0.125 & $\mathrm{~N}$ & \\
\hline 30 & 38 & 2 & & Y & & $\mathrm{N}$ & 0 & 0.699 & & 0.398 & -0.301 & Y & $\begin{array}{l}\text { IOP eleva- } \\
\text { tion }\end{array}$ \\
\hline 31 & 5 & 3 & & Y & & $\mathrm{N}$ & 1 & 2.301 & & 2.301 & 0.000 & $\mathrm{~N}$ & \\
\hline 32 & 4 & 4 & & Y & & N & 0 & 2.301 & & 2.301 & 0.000 & $\mathrm{~N}$ & \\
\hline 33 & 0 & 7 & & Y & & $\mathrm{N}$ & 0 & 0.699 & & 0.699 & 0.000 & Y & Hypotony \\
\hline 34 & 14 & 3 & & Y & & $\mathrm{N}$ & 0 & 1.301 & & 0.523 & -0.778 & $\mathrm{~N}$ & \\
\hline 35 & 18 & 5 & & Y & & $\mathrm{N}$ & 0 & 0.174 & & 0.000 & -0.174 & $\mathrm{~N}$ & \\
\hline 36 & 2 & 8 & & Y & & $\mathrm{N}$ & 1 & 2.602 & & 2.602 & 0.000 & Y & Hypotony \\
\hline 37 & 15 & 8 & & Y & & $\mathrm{N}$ & 1 & 1.854 & & 1.000 & -0.854 & $\mathrm{~N}$ & \\
\hline 38 & 12 & 1 & & Y & & $\mathrm{N}$ & 0 & 0.398 & & 0.260 & -0.138 & $\mathrm{~N}$ & \\
\hline 39 & 16 & 3 & & Y & & $\mathrm{N}$ & 0 & 0.456 & & 0.222 & -0.234 & $\mathrm{~N}$ & \\
\hline 40 & 8 & 6 & & Y & & $\mathrm{N}$ & 0 & 0.398 & & 0.301 & -0.097 & $\mathrm{~N}$ & \\
\hline 41 & 8 & 4 & & Y & & $\mathrm{N}$ & 0 & 0.481 & & 0.097 & -0.385 & $\mathrm{~N}$ & \\
\hline 42 & 13 & 4 & & Y & & N & 0 & 1.000 & & 1.000 & 0.000 & $\mathrm{~N}$ & \\
\hline 43 & 34 & 5 & & Y & & $\mathrm{N}$ & 0 & 1.000 & & 1.000 & 0.000 & Y & $\begin{array}{l}\text { IOP eleva- } \\
\text { tion }\end{array}$ \\
\hline 44 & 23 & 3 & & Y & & $\mathrm{N}$ & 0 & 0.301 & & 0.155 & -0.146 & $\mathrm{~N}$ & \\
\hline 45 & 10 & 3 & & Y & & $\mathrm{N}$ & 0 & 0.301 & & 0.301 & 0.000 & $\mathrm{~N}$ & \\
\hline 46 & 16 & 2 & & Y & & $\mathrm{N}$ & 0 & 0.155 & & 0.301 & 0.146 & $\mathrm{~N}$ & \\
\hline 47 & 8 & 5 & & Y & & $\mathrm{N}$ & 0 & 1.854 & & 1.301 & -0.553 & $\mathrm{~N}$ & \\
\hline 48 & 15 & 7 & & Y & & $\mathrm{N}$ & 0 & 0.000 & & 0.000 & 0.000 & $\mathrm{~N}$ & \\
\hline
\end{tabular}


Table 1 (continued)

\begin{tabular}{|c|c|c|c|c|c|c|c|c|c|c|}
\hline Patient & IOP Day 1 & $\begin{array}{l}\text { \#Times } \\
\text { ancillary Instrument } \\
\text { placed in eye }\end{array}$ & Objective achieved? & Wound leak? & Pain score (0-4) & Preoperative DCVA & Month 3 DCVA & logMAR Change & Adverse Events & Comments \\
\hline 49 & 11 & 2 & Y & N & 0 & 1.301 & 0.481 & -0.820 & N & \\
\hline 50 & 30 & 6 & Y & N & 0 & 1.301 & 0.301 & -1.000 & Y & $\begin{array}{l}\text { IOP eleva- } \\
\text { tion }\end{array}$ \\
\hline Mean & 12.0 & 4.5 & & & & 1.237 & 0.713 & -0.524 & & \\
\hline SD & 8.3 & 1.9 & & & & 0.881 & 0.656 & 0.562 & & \\
\hline
\end{tabular}

Abbreviations: AE Adverse Events, AM Amniotic membrane, DCVA Distance corrected visual acuity, DIS Dislocated Intraocular lens, ERM Epiretinal membrane, FAX Fluid air exchange, GT Gas tamponade, ILM Internal limiting membrane, LX Endolaser photocoagulation, INTRAOP Intraoperative, MD Membrane dissection, MH Macular hole, OR Operative, PPV Pars plana vitrectomy, PREOP Preoperative, RIOL Removal of dislocated IOL, RLF Retained lens fragments, RRD Rhegmatogenous retinal detachment, SIOL Secondary intraocular lens implantation, ST Silicone oil tamponade, TRD Traction retinal detachment, VH Vitreous hemorrhage, VIT Vitritis

across 3 follow-up visits and found statistically significant improvements in the 2 groups at each postoperative visit [8]. An approximately 10 letter gain was noted at 3 months among those undergoing 25G vitrectomy. A retrospective study of ERM surgeries by Naruse et al. also reported visual gains of $4.6 \pm 13.4$ letters in the third postoperative month when using $25 \mathrm{G}$ systems [9]. In 5 patients with isolated ERM, the average visual improvement in our study was approximately $4 \pm 4.18$ letters. Two studies compared the flat tip, $7500 \mathrm{~cm} 25 \mathrm{G}$ and $27 \mathrm{G}$ systems in cases of RRD [10,11]. Rizzo et al. reported an improvement of 30 letters 3 months after surgery while Sborgia et al. similarly reported an improvement of 35 letters. These were similar to our subset of 9 patients with a sole diagnosis of RRD who had an improvement of $19.22 \pm 15.10$ letters by the third postoperative month. A study by Naruse et al. reported an improvement of $17.5 \pm 28.1$ letters among patients operated on for proliferative diabetic retinopathy using $25 \mathrm{G}$ flat tip $5000 \mathrm{cpm}$ system [12]. In our subset of patients with vitreous hemorrhage and tractional retinal detachment, the improvement in vision in 3 months was $26.5 \pm 15.7$ ETDRS letters (Table 3).

The results of this study suggest that employment of the 25G BTCP may decrease operative times. We observed that mean and total operative times for individual phases of the PPV procedure were closer to the lower end of the ranges reported by similar studies using $25 \mathrm{G}$ probes (Table 4). Total operative time was observed to be correlated with the number of surgical steps and ancillary instruments used. These 3 closely-related variables indicating surgical complexity were uniquely quantified in this study. As longer operative durations and frequent instrument entry and exit may increase the risk for complications, new advances that shorten operating and recovery times, enhance surgeon productivity, and lower procedural costs are always welcome. We understand, however, that because case complexity and surgeon skill can independently influence operative time, a direct comparison of surgical efficiency across different practices and time periods is difficult and should be done with caution.

The BTCP features a port opening that is significantly closer to the distal tip (0.009 in.), half the distance of conventional flat-tip probes (0.018 in.). The multifunctional capabilities of this unique probe geometry have been supported by laboratory and clinical studies $[15,16]$. This shortened port-tip distance improves access to surgical tissue planes and facilitates aspiration of preretinal and subretinal materials and has the potential to improve surgical efficiency. With this working distance, an improved ability of the dual-pneumatic probe to control fluidics, and enhanced surgeon control via machine software and hardware, the BTCP can be used in a multifunctional role to perform many steps currently being done using ancillary instruments. For example, the BTCP tip can be used to grasp and manipulate pre-retinal and even thin internal limiting membranes thus minimizing the use of tissue forceps. The fine tip can be insinuated into preretinal membranes and be used as a scissors to nibble and cut fibrovascular tissues. We have also used the BTCP as a flute needle to aspirate fluid during complete fluid-air exchange. The BTCP tip can also be brought closer to the retinal surface to aspirate thick, coagulated heme and to minimize the need for refluxing fluid to blow off preretinal material. Fig. 2 shows how various surgical steps can be achieved by using the cutter probe alone (See Video 1 Supplemental Digital Content, Surgical Maneuvers). Several maneuvers such as the "lift-and-shave" and "shovel-and-cut" techniques have been described to enable surgeons to dissect diabetic membranes with greater facility which may also lessen the use of ancillary instruments $[16,17]$. The smaller $27 \mathrm{G}$ BTCP may further improve tissue access but may also decrease vitreous flow.

Although we were able to perform several maneuvers using the ultra-high speed BTCP in place of forceps, scissors and soft-tip cannulas, it should be emphasized that a beveled-tip cutter is not optimal for these steps and should not be used whenever more proper 
Table 2 Correlation Analysis of Surgical Variables

A. Number of Surgical Steps Ver
Total Operative Time
Number of Times Ancillary Instr
Postoperative Day 1 IOP
Shave Vitrectomy Time
Core+Shave Victrectomy Time
Core Vitrectomy Time
logMAR change
Total Operative Time

Postoperative Day 1 IOP

Core Vitrectomy Time

logMAR change

Shave VItrectomy Time

Core+Shave Victrectomy Time

C. Postoperative Day 1 IOP Versus Number of Surgical Steps

Total Operative Time

Number of Times Ancillary I trument Used

Shave Time

Core+Shave Victrectomy Time

Core Time

\section{Postoperative Pain Score Versus}

Core Vitrectomy Time

Number of Surgical Steps

Postoperative Day 1 IOP

Number of Times Ancillary I trument Used

Core+Shave Victrectomy Time

Total Operative Time

Shave Vitrectomy Time

logMAR change

E.Total Vitrectomy Time (Core + Shave) Versus

Total Operative Time

logMAR change

Number of Surgical Steps

Postoperative Day 1 IOP

Number of Times Ancillary I trument Used

Postoperative Pain Score

\section{F. logMAR change}

Total ORTIME

Shave Time

Core Time

PPV

Number of Steps

Number of instruments

Pain
B. Number of Times Ancillary Instrument Used Versus

\begin{tabular}{|c|c|}
\hline r value & pvalue \\
\hline 0.593 & $<0.0001 *$ \\
\hline 0.483 & $0.0004^{*}$ \\
\hline 0.293 & $0.0388^{*}$ \\
\hline 0.195 & 0.1757 \\
\hline 0.195 & 0.1757 \\
\hline 0.116 & 0.4241 \\
\hline 0.046 & 0.7532 \\
\hline Pearson $r$ & pvalue \\
\hline 0.535 & $0.0001 *$ \\
\hline-0.066 & 0.6476 \\
\hline 0.044 & 0.7599 \\
\hline 0.018 & 0.9006 \\
\hline-0.030 & 0.8362 \\
\hline-0.011 & 0.9397 \\
\hline rvalue & pvalue \\
\hline 0.293 & $0.0388^{*}$ \\
\hline 0.178 & 0.2158 \\
\hline-0.066 & 0.6476 \\
\hline 0.050 & 0.7325 \\
\hline 0.047 & 0.7469 \\
\hline 0.020 & 0.893 \\
\hline rvalue & $p$ value \\
\hline 0.156 & 0.2794 \\
\hline-0.153 & 0.2897 \\
\hline-0.137 & 0.3432 \\
\hline 0.093 & 0.5191 \\
\hline 0.0867 & 0.5492 \\
\hline-0.023 & 0.8734 \\
\hline-0.002 & 0.9914 \\
\hline-0.066 & 0.6497 \\
\hline$r$ value & pvalue \\
\hline 0.5608 & $0.0001 *$ \\
\hline 0.252 & 0.0773 \\
\hline 0.195 & 0.1757 \\
\hline 0.047 & 0.7469 \\
\hline-0.011 & 0.9397 \\
\hline-0.023 & 0.8734 \\
\hline rvalue & pvalue \\
\hline 0.234 & 0.1019 \\
\hline 0.224 & 0.1174 \\
\hline 0.223 & 0.1194 \\
\hline 0.252 & 0.0773 \\
\hline 0.046 & 0.7532 \\
\hline 0.018 & 0.9006 \\
\hline-0.066 & 0.6497 \\
\hline
\end{tabular}

* significant

Abbreviation: IOP Intraocular pressure 
Table 3 Comparison of 3-month postoperative visual acuities across different 25G studies

\begin{tabular}{|c|c|c|}
\hline Indication & Instrumentation & $\begin{array}{l}\text { Visual Acuity } \\
\text { Gain (ETDRS } \\
\text { Letters) }\end{array}$ \\
\hline \multirow[t]{3}{*}{ RRD } & $25 \mathrm{G}$, beveled tip, 10,000 cpm (Current Study, $n=9$ ) & $19.22 \pm 15.10$ \\
\hline & 25G, flat tip, 7500 cpm (Sborgia et al., 2019) [1 1] & 35 \\
\hline & 25G, flat tip, 7500 cpm (Rizzo et al., 2017) [10] & 30 \\
\hline \multirow[t]{3}{*}{ ERM } & $25 \mathrm{G}$, beveled tip, 10,000 cpm (Current Study, $n=5$ ) & $4 \pm 4.18$ \\
\hline & 25G, flat tip, 5000 cpm (Naruse et al., 2017) [9] & $4.6 \pm 13.4$ \\
\hline & 25G, flat tip, 5000 cpm (Mitsui et al., 2016) [8] & 10 \\
\hline \multirow[t]{2}{*}{ PDR } & 25G, beveled tip, 10,000 cpm (Current Study, $n=4$ ) & $26.50 \pm 15.70$ \\
\hline & 25G, flat tip, 5000 cpm (Naruse et al., 2019) [12] & $17.5 \pm 28.1$ \\
\hline
\end{tabular}

Legend: RRD Rhegmatogenous retinal detachment, ERM Epiretinal membrane, PDR Proliferative diabetic retinpoathy

Table 4 Comparison of total operative and vitrectomy times across different 25G studies

\begin{tabular}{|c|c|c|c|}
\hline Surgical Parameter & Indication & Instrumentation & Time (Minutes) \\
\hline \multirow[t]{5}{*}{ Total Operative Time } & RRD & 25G, beveled tip, 10,000 cpm (Current Study) & $39.0 \pm 14.2$ \\
\hline & & 25G, flat tip, 7500 cpm (Sborgia et al., 2019) [11] & $64.4 \pm 9.5$ \\
\hline & ERM & 25G, beveled tip, 10,000 cpm (Current Study) & $22.4 \pm 4.1$ \\
\hline & & 25G, flat tip, 5000 cpm (Naruse et al., 2017) [9] & $32.7 \pm 10.1$ \\
\hline & & 25G, flat tip, 5000 cpm (Mitsui et al., 2016) [8] & $16.1 \pm 9.3$ \\
\hline \multirow[t]{8}{*}{ Total Vitrectomy Time } & RRD & 25G, beveled tip, 10,000 cpm (Current Study) & $14.4 \pm 5$ \\
\hline & & 25G, flat tip, 7500 cpm (Sborgia et al., 2019) [11] & $20.8 \pm 3.8$ \\
\hline & & 25G, flat tip, 7500 cpm (Rizzo et al., 2017) [10] & $19.6 \pm 7.3$ \\
\hline & ERM & 25G, beveled tip, 10,000 cpm (Current Study) & $4.7 \pm 1.7$ \\
\hline & & 25G, flat tip, 5000 cpm (Mitsui et al., 2016) [8] & $6.2 \pm 2.7$ \\
\hline & Various Indications & 25G, beveled tip, $1000 \mathrm{cpm}$ (Current Study) & $8.9 \pm 6.5$ \\
\hline & & 25G, flat tip, 7500 cpm (Rizzo et al., 2011) [14] & $18.4 \pm 9.6$ \\
\hline & & 25G, flat tip, 5000 cpm (Rizzo et al., 2011) [14] & $26.4 \pm 14.6$ \\
\hline
\end{tabular}

Legend: RRD Rhegmatogenous retinal detachment, ERM Epiretinal membrane

instrumentation is available. Ancillary instruments are preferable for many surgical steps, such as retinal scissors for dissecting adherent or broad-based diabetic membranes and membranes on detached and atrophic retina, soft tip cannulas for FAX for a less traumatic aspiration over the macula and optic nerve head, and retinal forceps for initiating pinch-and-peel ERM and ILM peeling. The BTCP can however be used complementarily with a second instrument for manipulating and dissecting tissues, such as combining with a retinal pick to lift adherent posterior vitreous under chandelier lighting, or with retinal scissors for dissection of dense membranes. We find that a learning curve exists for utilizing BTCP in a multifunctional role.

We observed no additional safety concerns using the BTCP. Common intraoperative complications such as retinal and vitreous hemorrhage, iatrogenic breaks, and postoperative pressure changes occurred at a similar frequency as in previous reports [18-20]. These were readily addressed using conventional measures such as laser photocoagulation, IOP elevation, gas tamponade and postoperative medications. The incidence of immediate postoperative hypotony and IOP elevation were also similar to those reported in previous literature using conventional MIVS $[8-12,19,20]$. The use of a smaller $27 \mathrm{G}$ probe may mitigate the risks for postoperative hypotony in complex cases [21].

A recent meta-analysis has reported redetachment rates of about $20.9 \%$ after primary PPV [22]. Smaller gauge instrumentation with improved vitreous cutting and fluidics may minimize iatrogenic tears and postoperative RD by reducing pulsatile traction, wound leaks, vitreous-wound incarceration, iatrogenic retinal trauma, and facilitating pre-retinal traction membrane removal. Re-detachment occurred in 1 eye treated for RRD which was at a higher risk because of very long axial length. This was successfully treated with repeat PPV and silicone oil tamponade. No cases of endophthalmitis were observed 

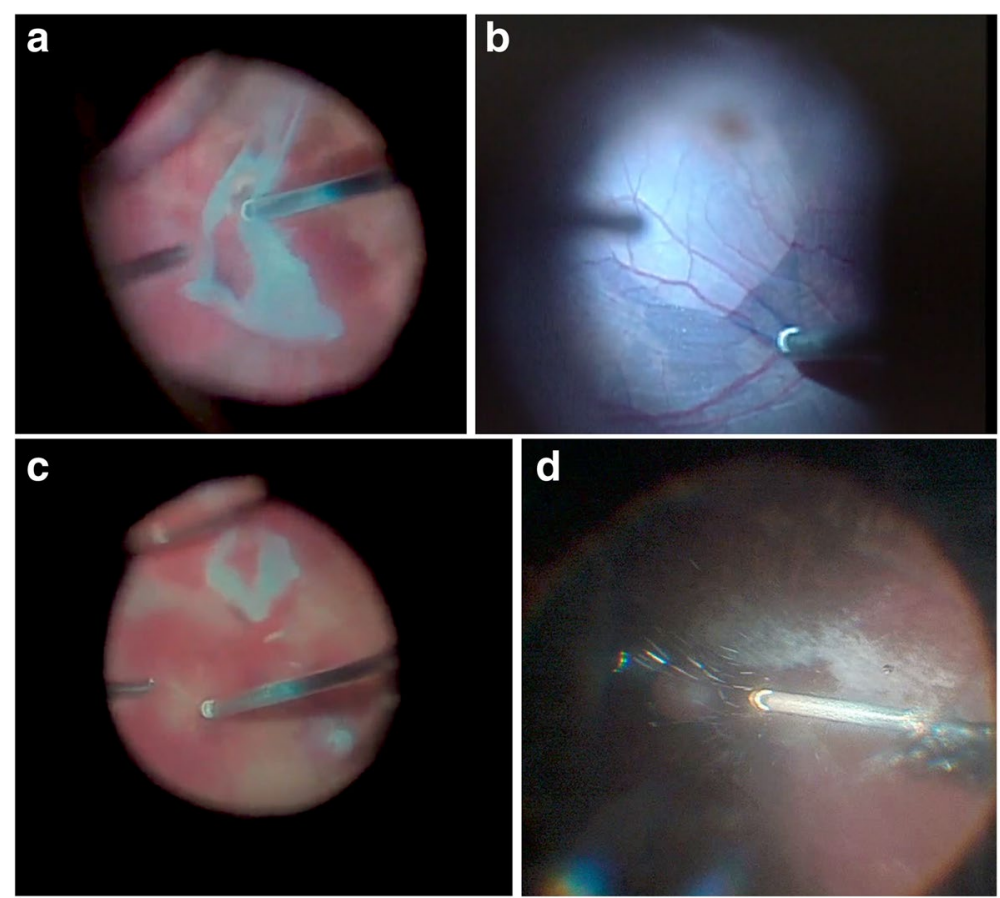

Fig. 2 Surgical microscope view of 25-gauge, beveled-tip probe utilized to engage pre-retinal fibroproliferative tissues during membrane dissection (A), to peel the internal limiting membrane and overlying epiretinal membrane after staining with a combination of Brilliant Blue and trypan blue dye (B), and to remove a thin layer of heme near the retinal surface using aspiration mode only (C). An even, laminar flow of triamcinolone acetonide-stained vitreous into the port-opening can be achieved using open-biased duty cycle (D)

in this series. The procedure was well tolerated by majority of patients who reported absence of pain within the first $24 \mathrm{~h}$ after surgery.

The results of this study lend evidence to the ability of ultra-high speed, $10,000 \mathrm{cpm}$ probes to shorten vitreous gel removal time. In vitro studies have reported a proportional relationship between vitreous flow and cutting speeds when using 50/50 or biased closed duty cycle across different cutter probe gauges $[23,24]$. The amount of aspirated vitreous collagen material is related to cutter characteristics as summarized in the equation:

Theoretical Vitreous Chunk Length=Flow Rate through Aspiration Line / (Cutter Port Surface x Cut Rate) [25].

By utilizing ultra-high cut rates, vitreous can be quickly segmented into smaller pieces facilitating smoother, less turbulent aspiration even when using smaller diameter lumen [24]. Higher cut rates can also enhance surgical precision and safety by minimizing pulsatile vitreous movement and avoiding iatrogenic retinal breaks.

Flow dynamic studies in porcine eyes have demonstrated faster aspiration and reflux velocities when using BTCP [15]. Beveled-tip geometry has been reported to prolong high aspirating pressures during the duty cycle and lower tip turbulence at the port opening [22]. The improved flow dynamics of the BTCP contribute to faster vitreous aspiration which may account for the shorter vitrectomy times observed in this study.

Our prospective study design included standardized measurement of efficacy and efficiency variables, such as operative times, number of surgical steps and ancillary instrument use. We also conducted correlation analysis to identify associations among variables and established a direct relationship between total operative time and number of ancillary instruments used and number of times such instruments were used. This indicates that for more complex cases, surgeons employ adjunctive instrumentation more frequently. Further research is therefore needed to explore whether new technologies, such as BTCP or other cutter probe configurations, that perform multiple functions may help decrease operative times and complications.

This study demonstrates the effectiveness of a BTCP for the surgical treatment of common VR indications in producing generally similar outcomes as those achieved using earlier cutter probe configurations. Furthermore, while acknowledging the limitations of comparing studies from different institutions and population groups, our results suggest that operative times may be reduced using a 25-gauge, $10,000 \mathrm{cpm}, \mathrm{BTCP}$ system. The TOT and TVT observed in this study, for a given indication, were shorter compared to the majority of studies wherein these 
temporal parameters have been reported (Table 4). Novel surgical parameters quantified in this study included the number of ancillary instruments used as well as the number of instrument exchanges performed. We believe similar structural and functional outcomes were achieved using shorter operative durations because of unique features of the BTCP. The shorter surgical durations may allow a practice to perform more procedures during a given day and increase efficiency. Reduction in ancillary instrumentation use may also result in cost savings for the practice or reduce the risk of infection brought about by frequent instrument exchanges and imperfect sterilization of reusable instrumentation.

It should be recognized that while the BTCP may substitute for other instrumentation, in many instances, traditional ancillary instrumentation is still optimal for a specific task and are preferentially used such as with initiation of and extensive membrane peeling, bimanual surgery, drainage of subretinal fluid, pre- and subretinal fluid injections, removal of subretinal removal of very large nuclear fragments or foreign objects.

Our study has several limitations which should be considered in analyzing our results. The study was descriptive in that we used only a single type of MIVS cutter probe without a control group which does not allow us to directly compare surgical results with other available probes, machines, and settings. Given the small patient population, our study is not sufficiently powered to detect rare events such as endophthalmitis, choroidal bleed, and subretinal migration of tamponade agents. The surgeries were done by 3 different vitreoretinal specialists to account for differences in surgical preferences and techniques, although we understand that our single center design may not be representative of the greater surgical community. A larger surgeon population should also be able to decrease potential data collection bias. Larger, randomized, controlled trials involving multiple surgeons are needed to compare the different probe designs. We recognize that operative duration and clinical outcomes are influenced not only by the type of MIVS utilized but also by other factors such as stage of the disease, operative complexity, patient cooperation, quality of surgical assistants and other operating room equipment such as surgical microscope and viewing systems. The results of this investigation provide basis for larger studies to fully examine the value of BTCP across a broad range of conditions. In conclusion, an ultra-high speed, 25G, BTCP appears effective and safe for treating a variety of VR conditions and has the potential to reduce the use of ancillary instrumentation and operative time in pars plana vitrectomy. Further studies are needed to fully elucidate the advantages and limitations of this novel probe design.

\section{Supplementary Information}

The online version contains supplementary material available at https://doi. org/10.1186/s12886-022-02311-3.

Additional file 1. Supplemental Digital Content. Video demonstrating 25-gauge, beveled tip cutter probe during diabetic retinopathy surgery.

\section{Acknowledgments}

Not applicable.

\section{Financial disclosures}

HSU has received speaker honoraria and research grants from Alcon Surgical. None of the other authors have any financial interests in the products mentioned in this article

\section{Authors' contributions}

HSU was responsible for the conception and design of the study. HSU, VLOC, PSC and JTF contributed to the data collection. Data analysis and interpretation were done by HSU, VLOC and JCMA. HSU, VLOC and JTF contributed to the writing of the manuscript, while HSU, VLOC, JCMA and PSC did critical substantive revision of the final manuscript. Figures and tables were prepared by HSU, VLOC and JCMA. All authors approved the final version of the manuscript and have agreed to be responsible for the work.

\section{Author's information}

Not applicable.

\section{Funding}

None.

\section{Availability of data and materials}

All data generated or analysed during this study are included in this published article.

\section{Declarations}

Ethics approval and consent to participate

The study was conducted according to the tenets of the Declaration of Helsinki. The study protocol and informed consent form was approved by the Peregrine Eye and Laser Institute (PELI) Institutional Review Board (Philippine Health Ethics Research Board Registration \# L3-2018-043-02, Metro Manila,

Philippines). Participants provided informed consent prior to enrollment.

Consent for publication

Not Applicable.

\section{Competing interests}

HSU has previously received speaker honoraria from Alcon Surgical Inc. The rest of the authors have no possible competing interests to disclose.

\section{Author details}

${ }_{1}^{1}$ Peregrine Eye and Laser Institute, Morning Star Center, 347 Gil Puyat Avenue, Bel Air, 1209 Makati City, Philippines. ${ }^{2}$ Department of Ophthalmology and Visual Sciences, University of the Philippines, Manila, Philippines. ${ }^{3}$ Eye Institute, St Luke's Medical Center, Quezon City, Philippines. ${ }^{4}$ Cebu Doctors' University Hospital, Cebu City, Philippines. ${ }^{5}$ Moorfields Eye Hospital NHS Foundation Trust, London, UK. ${ }^{6}$ Mary Mediatrix Medical Center, Lipa City, Philippines.

Received: 4 June 2021 Accepted: 10 February 2022

Published online: 24 February 2022

\section{References}

1. Machemer R, Buettner H, Norton E, Parel JM. Vitrectomy: a pars plana approach. Trans Am Acad Ophthalmol Otolaryngol. 1972;75(4):813-20.

2. Lin X, Apple D, Hu J, Tewari A. Advancements of vitreoretinal surgical machines. Curr Opin Ophthalmol. 2017;28(3):242-5. 
3. de Oliveira PRC, Berger AR, Chow DR. Vitreoretinal instruments: vitrectomy cutters, endoillumination and wide-angle viewing systems. Int J Retin Vitr. 2016;2:28.

4. González-Saldivar G, Chow DR. Update in Vitreoretinal Instrumentation. US Ophthalmic Rev. 2018;11(2):98-102.

5. Francisconi CLM, Chow DR. What's new in vitreoretinal instrumentation? New designs, cut rates, and port migration aim to increase efficiency. Retin Phys. 2018;15:34-7.

6. Fujii GY, De Juan E, Humayun MS, et al. A new 25-gauge instrument system for transconjunctival sutureless vitrectomy surgery. Ophthalmology. 2002;109(10):1807-12.

7. Oshima Y, Wakabayashi T, Sato T, et al. A 27-gauge Instrument system for transconjunctival sutureless microincision vitrectomy surgery. Ophthalmology. 2010;117:93-102.

8. Mitsui K, Kogo J, Takeda H, et al. Comparative study of 27-gauge vs 25-gauge vitrectomy for epiretinal membrane. Eye (Lond). 2016;30(4):538-44.

9. Naruse S, Shimada H, Mori R. 27-gauge and 25-gauge vitrectomy day surgery for idiopathic epiretinal membrane. BMC Ophthalmol. 2017;17(1):188

10. Rizzo S, Polizzi S, Barca F, et al. Comparative study of 27-gauge versus 25-gauge vitrectomy for the treatment of primary Rhegmatogenous retinal detachment. J Ophthalmol. 2017;2017:6384985.

11. Sborgia G, Niro A, Sborgia L, et al. One-year outcomes of 27-gauge versus 25-gauge pars plana vitrectomy for uncomplicated rhegmatogenous retinal detachment repair. Int J Retina Vitreous. 2019;4(5):13.

12. Naruse Z, Shimada H, Mori R. Surgical outcomes of 27-gauge and 25-gauge vitrectomy day surgery for proliferative diabetic retinopathy. Int Ophthalmol. 2019;39(9):1973-80.

13. Mori R, Naruse S, Shimada H. Comparative study of 27-gauge and 25-gauge vitrectomy performed as day surgery. Int Ophthalmol. 2018;38(4):1575-82.

14. Rizzo S, Genovesi-Ebert F, Belting C. Comparative study between a standard 25-gauge vitrectomy system and a new ultrahigh-speed 25-gauge system with duty cycle control in the treatment of various vitreoretinal diseases. Retina. 2011;31(10):2007-13.

15. Inoue M, Koto T, Hirakata A. Flow dynamics of beveled-tip and flat-tip vitreous cutters. Retina. 2021;41(2):445-53.

16. González-Saldivar G, Chow DR. The Shovel and cut technique: Beveled vitrectomy probes to address diabetic tractional retinal detachments. Retina. 2020.

17. Berrocal MH. All-probe vitrectomy dissection techniques for diabetic tractional retinal detachments: lift and shave. Retina. 2018;38(Suppl 1):S2-4.

18. Mariotti C, Nicolai M, Saitta A, et al. Standard cut rate 25-gauge vitrectomy versus ultra-high speed 25 -gauge system in core vitrectomy: a randomized clinical trial. Retina. 2016;36(7):1271-4.

19. Rizzo S, Belting C, Genovesi-Ebert F, di Bartolo E. Incidence of retinal detachment after small-incision, sutureless pars plana vitrectomy compared with conventional 20-gauge vitrectomy in macular hole and epiretinal membrane surgery. Retina. 2010;30(7):1065-71.

20. Ahn SJ, Woo SJ, Ahn J, Park KH. Comparison of postoperative intraocular pressure changes between 23-gauge transconjunctival sutureless vitrectomy and conventional 20-gauge vitrectomy. Eye (Lond). 2012;26(6):796-802.

21. Charles S, Ho AC, Dugel PU, et al. Clinical comparison of 27-gauge and 23-gauge instruments on the outcomes of pars plana vitrectomy surgery for the treatment of vitreoretinal diseases. Curr Opin Ophthalmol. 2020;31(3):185-91.

22. Znaor L, Medic A, Binder S, et al. Pars plana vitrectomy versus scleral buckling for repairing simple rhegmatogenous retinal detachments. Cochrane Database Syst Rev. 2019;3(3):CD009562.

23. Dugel PU, Abulon DJ, Dimalanta R. Comparison of attraction capabilities associated with high-speed, dual-pneumatic vitrectomy probes. Retina. 2015;35(5):915-20.

24. Abulon DJ, Buboltz DC. Porcine vitreous flow behavior during high speed vitrectomy up to 7500 cuts per minute. Trans Vis Sci Technol. 2016;5(1):1-5.

25. Rossi T, Querzoli G, Angelini G, Malvasi C, Rossi A, Morini M, et al. Hydraulic resistance of vitreous cutters: the impact of blade design and cut rate. TransI Vis Sci Technol. 2016;5:1-11.

\section{Publisher's Note}

Springer Nature remains neutral with regard to jurisdictional claims in published maps and institutional affiliations.

Ready to submit your research? Choose BMC and benefit from:

- fast, convenient online submission

- thorough peer review by experienced researchers in your field

- rapid publication on acceptance

- support for research data, including large and complex data types

- gold Open Access which fosters wider collaboration and increased citations

- maximum visibility for your research: over $100 \mathrm{M}$ website views per year

At BMC, research is always in progress.

Learn more biomedcentral.com/submissions 